\section{Improving attendance in outpatient clinics}

Sir - The study reported by Munjal and colleagues in the Irish Journal of Psychological Medicine (11: 182-4) suggests that the process of asking families to confirm child mental health appointments using questionnaire and stamped addressed envelope (sae) may improve attendance. We set out to see whether their scheme was cost effective in routine clinical practice and also to compare the use of such questionnaires to simple appointment letters (which they did not do). A questionnaire (with s.a.e) was sent to every alternate referred child mental health patient. It invited one of three responses: i) will attend, ii) want a different appointment date, and iii) no longer require an appointment. A design with alternate letters was favoured to random allocation which would have been less likely to control for shifts in waiting time and the effects of weather variations on attendance. The study was continued for a full calendar year collecting information in a similar way to Munjal and colleagues.

Over the year 137 new patient referrals were received. Of these, 99 were from general practitioners, 14 from paediatricians and community medical officers, 11 from other psychiatric consultants, and the remaining 13 from psychologists, community psychiatric nurses and educational welfare officers. There was an average of 27 days between the date of receipt of the referral to the date of the first appointment, with a range of 6-61 days. There were no significant differences between the two groups in terms of broad age groups, sex, source of referral or length of wait.

Questionnaires were sent to 69 newly referred patients. Eight were not returned, 54 replied to confirm attendance and 7 to cancel. Six of those cancelling requested a new date and 1 indicated that an appointment was no longer required. Overall, 92(67\%) of the 137 patients attended a first appointment; a further $23(17 \%)$ cancelled the appointment, and $22(16 \%)$ did not attend (DNA).

Receipt of the questionnaire significantly affected attendance at the first appointment. Of the questionnaire group $53(77 \%)$ attended their first appointment while $39(57 \%)$ of the 68 in the non-questionnaire group attended. This was a significant difference ( $p=0.013$ ).

Forty five patients did not attend the first appointment and of these 20 attended a subsequent appointment. Eight out of $16(50 \%)$ in the questionnaire group attended subsequently, and 12 of the $29(41 \%)$ in the non-questionnaire group attended a subsequent assessment (no significant difference). Overall $112(82 \%)$ patients attended at some stage after referral, $61(88 \%)$ from the questionnaire group and $51(75 \%)$ of the other group ever attended $(p=0.043)$. Sixty one patients returned the questionnaire and $50(82 \%)$ of these attended an assessment. Only three $(37 \%)$ of the non-returners attended ( $p=0.01$ ).

Previous studies have sought to explore why patients do not attend child mental health clinics ${ }^{1,2}$ and the interactive effects of factors such as age, sex, maternal employment and marital status have been explored. ${ }^{3}$ This study confined itself to the more pragmatic concern of improving attendance. The cost of running the trial involved a very small amount of extra secretarial time and the $£ 13.80$ spent on stamps. It was easy to set up and run. At the very least it merits a one year trial within teams to assess usefulnęss. We found that it reduced non-attendance without substantial extra cost.

References

1. Cottrell D, Hill P, Walk D, Dearnley J, Ierotheou A. Factors influencing non attendance at child psychiatry out-patient appointments. Br J Psychiat 1988; 152: 201-14.

2, Gould MS, Shaffer D, Kaplan D. The characteristics of dropouts form a child psychiatry clinic. J Am Acad Child Psychiat 1985; 24: 316-28.

3. Dowdney L, Rogers C, Dunn G. Influences upon attendance at out-patient facilities - the contribution of linear logistic modelling. Psychol Med 1993; 23: 195-201.

David West, Audit Officer Academic Unit of Child mental health, 12. A Clarendon Road, Leeds LS2 9NN, England.

*Barry Wright, MRCPsych, MRCGP, DCH, MMedSc, Consultant Child and family Psychiatrist, Lime. Trees Child and Family Unit, 31 Shipton Road, York YO3 6RE, England.

\section{Gastrointestinal presentations of Munchausen's syndrome: three case reports}

Sir - The syndrome of factitious disorders with physical symptoms was named Munchausen's syndrome by Richard Asher in 1951, after the eponymous dramatic, well travelled Baron.'

The principle features are uncontrollable pathological lying, with the presentation of the history in a dramatic, vague and inconsistent manner; evidence of prior treatments, including extensive hospital records and multiple scars; medical sophistication; disruptive hospitalisation, where the rules and regulations of the hospital are openly flaunted; symptoms that shift from one organ system to another; tolerance of painful and invasive procedures without complaint; demands for analgesic medication without signs of withdrawal on discontinuation and either the absence of visitors or one visitor who colludes in the behaviour. ${ }^{2-4}$ There is often an inordinate desire for privacy. Symptoms are not in keeping with the results of examination, blood tests or diagnostic procedures.

As is to be expected, these patients are diagnosed only after a series of investigations where no abnormality is detected and as such represent a significant drain on hospital resources. Subjects known to one medical institution frequently present at another, where a similar investigative procedure is conducted.

It appears from the literature that the incidence of Munchausen's syndrome is unknown. There is a justifiable reluctance to diagnose the condition, lest it deprive a patient of his/her right to treatment for a serious medical condition. In addition, there is an extensive use of aliases by these patients.

We report a series of three cases which presented in an urban general hospital within an eight month period, all 
on GI wards. The cases described each represent after a fashion, the stages of progression of behaviour seen in Munchausens patients.

\section{Case one}

The psychiatric team was asked to review a 19 year old male who was admitted post overdose of sedatives. The overdose was taken in front of a family member, with no suicidal intent and there was no evidence of a mental illness. Severe abdominal pain was given as motivation. The patient was a vague historian and stated that his abdominal pain prevented him from recalling details of his history. He was well versed in Crohn's disease and used many medical terms. He was in a private room which was devoid of cards, flowers etc. He claimed to have played several sports at an international level prior to his illness as outlined below and described himself as outgoing and extremely popular. He stated he would prefer if his family were not interviewed in order to spare them distress.

The patient had first presented 18 months previously with abdominal pain and frank blood PR. All investigations at this time were normal, including colonoscopy with biopsy, barium enema and Meckel's scan. Over the following 18 months, the patient had 13 accident and emergency attendances and eight hospital admissions, amounting to 31 weeks of inpatient care. He underwent a laparotomy and an ileostomy. The latter was performed for presumed Crohn's disease with non-specific pathology. A further admission for exploration of a non-functioning ileostomy revealed an orange segment in the stoma. Immediately prior to this admission, the subject had presented to another hospital in a different city with haematuria; an IVP revealed no abnormality.

During the present admission, during which time only his mother visited him, the patient continued to complain of abdominal pain for which no organic cause could be found. He persistently requested analgesia. When this was refused, he began complaining of pain and bleeding PR late at night, thereby obtaining medication from the duty doctors. He was commenced on total parenteral nutrition and a central line inserted. On several occasions he was noted to be eating chocolates despite his nil by mouth status. He was seen to be obtaining food from his mother and was quite open about this. A member of the nursing staff witnessed him stabbing his tongue and fingertips with a needle. The patient later denied this.

At no time was there evidence of a mental illness. The patient was discharged without confrontation following a further exploratory laparotomy.

\section{Case two}

This patient was a 32 year old man on whom a psychiatric opinion was requested regarding his refusal to eat. He had been admitted five weeks previously with bleeding PR and chest pain. His physical condition had been stable for the preceding fortnight but he had been refusing to eat. He had given his consent for nasogastric feeding but was removing the tube on a regular basis. He stated a motivation for discharge, although he accepted that his refusal to eat was precluding this. He stated that he needed surgical intervention in order to recover and explained the proposed operation in detail. He did not fulfil diagnostic criteria for anorexia nervosa or any other eating disorder or mental illness. It was not possible to interview any family member as the patient stated they had no telephone and were in the process of moving house and he could not recall the new address. No family member or friend visited him while he was in hospital.

Previous history was vague as the patient stated he could not recall the names of any of his doctors, nor could he recall the hospitals he had been treated in. His home address was nearly 150 miles from the hospital. He first presented two years previously with chest pain. Since that time he has attended $A \& E$ on numerous occasions and has had seven hospital admissions, amounting to a total of nine months of inpatient care. His first admission was with chest pain. All investigations including Echo, Holter and ECGs during attacks of pain were normal. Subsequent admissions were for infection of the left cubital fossa on two occasions and for PR bleeding on five occasions. He constantly sought analgesia. On each admission he had an iron deficiency anaemia and required transfusion. Surgical and haematological opinions were sought and numerous investigations carried out, including several colonoscopies, Meckel's scan, coeliac angiograms, red cell scan, haemolytic screen, bone marrow biopsy, laparoscopy and laparotomy. The only abnormality was third degree haentorrhoids which were identified as a bleeding point. These were injected twice but copious bleeding recurred almost immediately.

It came to light that in the previous five years this man had previously presented to his local hospital with pneumonia, bleeding PR with iron deficiency anaemia, haematemesis, melaena and chest pain.

During the present admission it was put to the patient that there was a suspicion that his symptoms and signs were being self-induced. He neither accepted nor denied this. However, immediately following this interview, he became quite angry and discharged himself from hospital. He. has not re-presented.

\section{Case three}

A 33 year old man from the UK was admitted via $A \& E$ with pneumaturia, pyrexia and a fistula discharging onto the anterior abdominal wall. He had been discharged two days previously from another hospital in Dublin following closure of a 'burst wound' which he claimed had now reopened. He had multiple abdominal wounds. He was an extremely vague historian who gave names and telephone numbers for friends and relatives subsequently found to be false. He stated that he was a professional footballer whose career had been cut short by recurrent medical problems. Eventually he gave the name of one hospital in the UK where he had received treatment. This hospital was contacted and his history obtained.

He had first presented eight years previously (1987) with abdominal pain. An appendicectomy was performed and histology was normal. Over the next three years he presented with several episodes of deliberate self-poisoning. He additionally had two admissions with abdominal pain and bloody diarrhoea. On each occasion colonoscopy was normal apart from non-specific inflammatory changes. In 1992 he presented at a second hospital with perforation of the small bowel which it was thought the patient had self-inflicted with a knife. Over the next year he had seven hospital admissions following self-induced opening of his abdominal wounds. Ultimately, a wire grille was inserted in an effort to prevent further self-harm. In May of 1995, the patient presented to a third hospital in the UK for closure of an ileostomy. It was this wound he 
had re-opened prior to his first presentation in Dublin.

During his admission, this man was extremely manipulative. He constantly demanded analgesia, stating he would take his discharge if he was refused. He was openly non-compliant with ward routine. He continuously removed his stoma bag, pulled out his central line and his TPN line. He was observed drinking from the tap in his room despite instructions to take nothing by mouth. He had substantial medical knowledge and claimed "anaphylactic reactions to non-steroidal anti-inflammatories and phenothiazinesn." He stated he would not "settle" on a ward and would continue to be disruptive unless moved to a private room. At one point he had a series of seizures following iv. administration of a drug. EEG and serum prolactin levels were normal. The patient stated he had fitted as a result of the antiemetic he was given and that he had warned the staff of this possibility. The medication was in fact an antibiotic which he had been receiving routinely. During his stay he developed a septicaemia. Enterococcus faecium grew from blood cultures.

Shortly after admission he admitted that he had deliberately re-opened his abdominal wound in order to gain admission to hospital. As he deteriorated he gave genuine contact numbers for relatives. He was unfit for surgery and was transferred back to the UK when medically stable. He is since deceased.

\section{Discussion}

Munchausen's syndrome is almost necessarily a diagnosis based on exclusion when exhaustive and expensive procedures fail to account for the patient's symptomatology.

There are three other reports of Munchausen's relating to inflammatory bowel disease. One reports a patient undergoing a total proctocolectomy with ileostomy for presumed ulcerative colitis caused by repeated laceration of the distal colon with a knitting needle. ${ }^{5}$ The second describes a patient undergoing distal sigmoid resection and colostomy for treatment of presumed indeterminate colitis caused by colonic injection of a caustic soda. ${ }^{6}$ The third describes a patient who presented with pseudo Crohn's disease, feigning rectal bleeding by injecting blood from her central line PR and inducing septicaemia by injecting faecal material into her central line.?

The three cases we describe vary in the severity of the illness inducing behaviour. Of the three, only the last admitted to self-infliction of his injuries, and this only when seriously ill. Suspicion may be aroused by the clinical features outlined in the introduction. However, this may not be enough to make a diagnosis of Munchausen's and the patient may either deny self-infliction or, more frequently abscond when confronted.

As mentioned above, the incidence of Munchausen's is uncertain. The above cases all presented to the same hospital within a relatively short time frame. However, even assuming that this is not a representative sample and the number of afflicted patients could be very small, the costs to the health service are significant. The nature of the condition and the lack of continuity between different services is of considerable danger to the patients. The keeping of a central Munchausen register has long been proposed and much debated. ${ }^{8-10}$ At present there remains no formal register in either the United Kingdom or the Republic of Ireland. There are obvious ethical issues surrounding such a register, not least that of physician patient confidentiality. Concerns have been expressed that such registers are not only ineffective but are likely to be used in a punitive and cathartic manner. ${ }^{10}$ Although there are informal local registers in current use, to our knowledge their efficacy or otherwise has not been demonstrated. We would suggest that a Munchausen register would serve both to decrease health service costs and to optimise the medical and psychological care of this group of patients.

References

1. Asher R. Munchausen's syndrome. Lancet 1951; i: 339-41.

2. Enoch MD, Trethowen WH. Uncommon Psychiatric Syndromes, Bristol: John Wright \& Sons, 1979.

3. Have GL, Jordan HW, Lockert EW. Munchausen's syndrome or chronic factitious illness: a review and case presentation. I Nat Med Assoc 1983; 75 : $175-81$.

4. Hyler SE, Sassman N. Chronic factitious disorder with physical symptoms (the Munchausen syndrome). Psychiatr Clin North Am 1981; 4: 365-77. 5. Daily WJR, Coles JH, Creger WP. Factitious anaemia. Ann Intern Med 1963; 58: 533-8.

6. Byrne JS, Carney MWP, Harry DS et al. Self-induced colitis. Postgrad Med J $1975 ; 51: 246-9$

7. Klein H, Du Vall AG, Klein S. Pseudo-Crohn's disease with bloody diarrhoea. Digestive Diseases and Sciences 1995; 40: 226-8.

8. Birch CA. Lancet 1951; i: 412.

9. Davey RI. A register of Munchausen cases. Psychiat Bull 1991; 15: 701.

10. Powell R, Boast N. The Million Dollar Man. Resource implications for chronic Munchausen's syndrome. Br J Psychiatry 1993; 162: 253-6.

Karen O' Flynn MB, MRCPsych, Lecturer in Psychiatry,

Alan O’ Donohoe MB, MRCPsych, Registrar in Psychiatry,

Michael Gill MD, MRCPsych, Senior Lecturer in Psychiatry, Trinity College Medical School, St. James's Hospital, Dublin 8, Ireland.

\section{Correction}

Re: Diogenes' syndrome - A load of old rubbish 1997; 14(3): 99-102

The paper stated that Dr Lynne Drummond was the first author. This was incorrect, the correct order of authors is as follows; Dr J Turner, Dr S Reid \& Dr L Drummond.

Our apologies for any inconvenience this may have caused to any of the authors. 